Communication

\title{
Transfer Hydrogenation Employing Ethylene Diamine Bisborane in Water and Pd- and Ru-Nanoparticles in Ionic Liquids
}

\author{
Sebastian Sahler, Martin Scott, Christian Gedig and Martin H. G. Prechtl * \\ Department of Chemistry, University of Cologne, Greinstrasse 6, 50939 Cologne, Germany; \\ E-Mails: ssahler@hiden.co.uk (S.S.); scott@itmc.rwth-aachen.de (M.S.); \\ cgedig@smail.uni-koeln.de (C.G.) \\ * Author to whom correspondence should be addressed; E-Mail: Martin.Prechtl@uni-koeln.de; \\ Tel.: +49-221-4701-981; Fax: +49-221-4701-788.
}

Academic Editor: Nicola Cioffi

Received: 2 April 2015 / Accepted: 8 September 2015 / Published: 17 September 2015

\begin{abstract}
Herein we demonstrate the use of ethylenediamine bisborane (EDAB) as a suitable hydrogen source for transfer hydrogenation reactions on $\mathrm{C}-\mathrm{C}$ double bonds mediated by metal nanoparticles. Moreover, EDAB also acts as a reducing agent for carbonyl functionalities in water under metal-free conditions.
\end{abstract}

Keywords: ruthenium; palladium; transfer-hydrogenation; ethylene diamine bisborane; ammonia borane, EDAB, reducing, carbonyl

\section{Introduction}

Hydrogenation is one of the most important chemical transformations used in academia and industry and has received notable attention in the past century [1-7]. It can be performed via transition metal-catalyzed activation of molecular hydrogen and is used in large-scale applications such as hydrocracking [8,9] and the Haber-Bosch-Process [10] or in medium- to lab-scale applications for the synthesis of fine and special chemicals [1-3,11]. However, as the use of molecular hydrogen often requires harsh reaction conditions [12] and the regio- and stereo-selectivity are challenging to control $[4,5,7,13]$, transfer hydrogenation has evolved as useful tool for specific and highly selective hydrogenation under mild conditions $[3,4,7,12,14,15]$. Specifically, the use of alcohols such as ethanol, 
isopropanol, and glycerol [4,7,16,17] and also compounds like formic acid [18] or Hantzsch's ester [19] have been used as suitable hydrogen sources in transfer hydrogenation reactions. Many of these transformations can be performed by using homogenous catalysis with transition metal complexes based on iron, palladium, ruthenium, or rhodium [4,7,15,17,20,21]. In contrast, the use of non-metal based organo-catalysts has also been shown and several prolin-based catalysts are known [22,23]. A major drawback of using alcohols or other carbon-based compounds in transfer hydrogenation lies in the comparable low mass fraction of the stored hydrogen volume-to-mass ratio. For example, isopropanol delivers only one equivalent $\left(3.3 \mathrm{wt}-\% \mathrm{H}_{2}\right)$ and formic acid $\left(4.4 \mathrm{wt}-\% \mathrm{H}_{2}\right)$ as well. Therefore, hydrogen-rich sources for transfer hydrogenation reactions are desirable. On the other hand, amine boranes such as ammonia borane $(\mathrm{AB})\left(\mathrm{NH}_{3} \mathrm{BH}_{3}\right)$ [24], methyl amine borane [24,25] (MAB), and ethylene diamine bisborane [24,26] (EDAB) have received increasing attention as hydrogen storage materials owing their hydrogen content. The mass fraction of stored hydrogen is comparably high and the compounds are easily obtained [24]. MAB releases a mass fraction of $9 \% \mathrm{H}_{2}$ [25,27], EDAB a mass fraction of $10 \%$ [26], and $\mathrm{AB}$ a mass fraction of up to $16 \% \mathrm{H}_{2}$ at a maximum temperature of $200{ }^{\circ} \mathrm{C}$ [24]. Amine boranes have not only been used as possible hydrogen storage material. Additionally, these non-toxic and water-soluble compounds have shown successful use as hydrogen sources for transfer hydrogenation reactions. $\mathrm{AB}$ in particular has been used elaborately for the reduction of $\mathrm{C}=\mathrm{C}$ [28], $\mathrm{C}=\mathrm{O}[29], \mathrm{C}=\mathrm{N}[13,30]$, or $\mathrm{NO}_{2}$ [13], thereby showing chemo-selectivity [13]. However, for other amine boranes, the literature is scant and little is known about chemo-, regio-, and stereo-selectivity. Our group has investigated the liberation of $\mathrm{H}_{2}$ from EDAB in ionic liquid media as a potential hydrogen storage material [26,31]. Following these studies, we focus on the use of EDAB as a possible hydrogen source for transfer hydrogenation reactions.

Referring to the ongoing debate of implementing greener processes in chemistry [32,33], but also regarding excellent behavior in catalytic conversions [34], the use of tailor-made ionic liquid media as reaction mediums has revealed encouraging effects for dehydrogenation reactions with amine boranes [26,31]. The incorporation of homogeneously dispersed metal nanoparticles as highly potent catalysts has also been demonstrated [31,35-41]. Furthermore, the use of nanoparticles as catalysts for the hydrolytic dehydrogenation of $\mathrm{AB}$ has been shown [42-45]. Additionally, EDAB undergoes hydrolytic dehydrogenation with elevated hydrogen yields compared to the reaction in common organic solvents if ionic liquids (ILs) are implemented as reaction medium. Subsequently, transition metal nanoparticles have been used as catalysts in transfer hydrogenation reactions, reducing $\mathrm{C}=\mathrm{C}$ [46] or $\mathrm{C}=\mathrm{O}$ [47] functionalities. However, to the best of our knowledge, the use of nanoparticles as transfer hydrogenation catalysts in ILs as reaction media has not yet been reported. Furthermore, the use of $\mathrm{EDAB}$ as a suitable hydrogen source in transfer hydrogenation reactions is also unknown.

\section{Results and Discussion}

Herein we present the use of EDAB as a hydrogen source as well as the use of Pd- and Ru-NPs (NPs: nanoparticles) as suitable transfer hydrogenation catalysts for the chemo-selective hydrogenation of carbonyl functionalities and for the reduction of unsaturated carbon-carbon bonds (Scheme 1). 


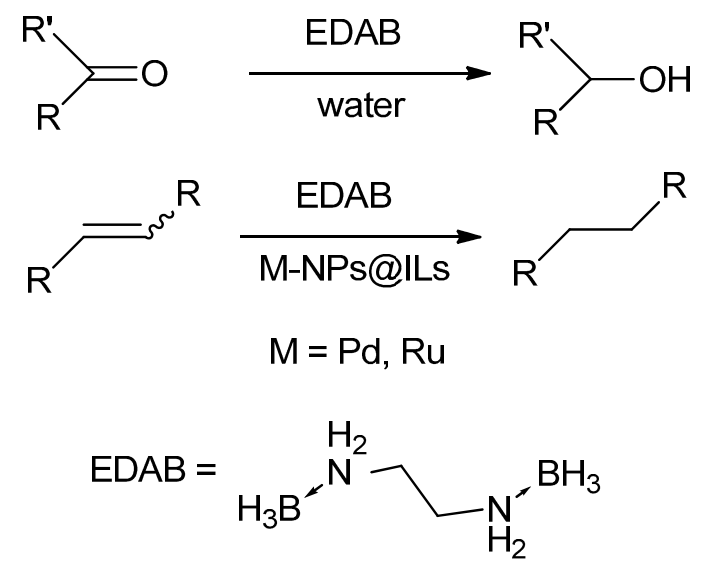

Scheme 1. EDAB as a hydrogen source for the reduction of carbonyl functionalities and unsaturated carbon-carbon bonds.

The application of amine borane adducts as highly active bench-top reducing agents has long been known $[48,49]$, but has fallen out of favor, and recently it has been demonstrated that they are suitable for safe reduction in pure water [50]. The reduction of carbonyl groups in different chemical surroundings is possible employing EDAB at mild temperatures and water as the solvent (Table 1).

Cyclohexanone (Table 1, entry 1 ) is quantitatively reduced by EDAB. The reduction of other simple carbonyl compounds like acetophenone (Table 1, entry 2), cyclohex-2-enone (Table 1, entry 3), and benzaldehyde (Table 1, entry 4) succeeds with moderate yields. While the aromatic substrates remain intact, the reduction of $\alpha, \beta$-unsaturated cyclohex-2-enone yields a mixture of the unsaturated cyclohex-2-enol (50\% yield) and the saturated cyclohexanol (13\% yield). Interestingly, the reduction of cinnamic aldehyde (Table 1, entry 5) yields solely the unsaturated cinnamic alcohol. The double bond remains unchanged, probably due to the stabilizing effect of the conjugated aromatic system. The comparison of the aliphatic carbonyl substrates octanal (Table 1, entry 6) and octan-3-one (Table 1, entry 7) reveals that aldehydes are by far more reactive toward EDAB than similar ketones. While octanal is reduced in 33\%-38\% yield after $1 \mathrm{~h}$, only $9 \%$ of octan-3-one reacts in the same time. The yield can be notably enhanced by prolonging the reaction time to $24 \mathrm{~h}(53 \%)$. The direct comparison between octanal and 3-octanone showed that 38\% 1-octanol and 9\% 3-octanol are produced. A similar behavior is observed when benzaldehyde and acetophenone are reduced in direct comparison: $93 \%$ phenylmethanol and $68 \%$ 1-phenylethanol are produced. These experiments indicate that the reduction of aldehydes is faster than ketones under the given conditions.

The reduction of benzophenone yields diphenylmethanol in moderate amounts $(48 \%-53 \%$; Table 1 , entry 8), while benzoquinone does not react under the given conditions (Table 1, entry 9). In contrast to the reduction of octan-2-one, the reduction of octan-2, 3-dione (Table 1, entry 10) yields moderate octan-2, 3-diol amounts $(37 \%-42 \%)$. The improved reactivity might be related to the superior solubility of the dione in comparison to the single ketone. Reduction of 5-hydroxymethylfurfural (HMF) also shows only a slow conversion into furandimethanol (Table 1 , entry $11 ; 10 \%$ ). Several other functional groups are not reduced by EDAB: alkenes, aromatic double bonds, nitro groups, lactames, esters, ethers, and acids remain inert, making EDAB highly chemo-selective for the reduction of aldehydes and ketones (Table 1, entry 12-16). The main influences on the yields of the reductions appear to be the solubility and the steric hindrance of the substrate. 
Table 1. Metal-free reductions using EDAB at $80{ }^{\circ} \mathrm{C}$ in $\mathrm{H}_{2} \mathrm{O}$.

(2)

a The yield was determined by ${ }^{1} \mathrm{H}-\mathrm{NMR}$ using $\mathrm{Si}_{2} \mathrm{Me}_{6}$ as internal standard. Each reaction was performed at least twice with a reaction time of $1 \mathrm{~h} ;{ }^{\mathrm{b}} 13 \%$ of cyclohexanol was found; ${ }^{\mathrm{c}}$ after $60 \mathrm{~min}$ and $24 \mathrm{~h}$, respectively.

In comparison to the reduction employing ammonia borane $(\mathrm{AB})$ or methyl amine borane (MAB) [50,51], reduction by EDAB is slightly slower, even at higher temperatures. The scope of substrates is very similar for all three reducing agents; a plethora of different carbonyl compounds are susceptible to reduction, leaving nearly all other functional groups intact. 
Besides the reduction of carbonyl compounds with neat EDAB in water, the application of metal nanoparticles as catalysts broadens the substrate scope considerably. Ruthenium and palladium were chosen as catalysis. These metals in particular are known to be active in hydrogenation and dehydrogenation reactions in ionic liquid media [31,35-37,39,41]. Additionally, ionic liquids were employed due to their stabilizing effect on the nanoparticles as well as the ionic solvent properties, which provide a good solubilization of the substrates. We evaluated the well-characterized nanoparticle catalysts $\mathrm{Ru} @[\mathrm{BMMIM}]\left[\mathrm{NTf}_{2}\right]$ (BMMIM: 1-n-butyl-2,3-dimethylimidazolium) and Pd@[(BCN)MIM][NTf $]$ ((BCN)MIM: 1-butyronitrile-3-methylimidazolium) [35,39] for transfer hydrogenation with EDAB as the hydrogen source. The results for the ruthenium-catalyzed reactions are listed in Table 2.

Table 2. Catalyzed transfer hydrogenations with EDAB and Ru@[BMMIM] $\left[\mathrm{NTf}_{2}\right]$ at $70{ }^{\circ} \mathrm{C}$ after $18-24 \mathrm{~h}$.

\begin{tabular}{ccccc}
\hline$\#$ & Substrate & {$[\%]^{\mathbf{a}}$} & {$[\%]$ Overall } & TON $^{\mathbf{b}}$ \\
\hline 1 & cyclohexene & 25 & 25 & $72(137)$ \\
2 & 1,5-cyclooctadiene & $55(5)$ & 60 & $133(253)$ \\
3 & benzonitrile & 0.0 & 0 & 0.0 \\
4 & nitrobenzene & $0.0(17)$ & 17 & $108(205)$ \\
5 & phenylacetylene & $8.1(7.7)$ & 16 & $50(95)$ \\
6 & diphenylacetylene & $22(5)$ & 27 & $45(86)$ \\
7 & styrene & 45 & 45 & $127(241)$ \\
8 & squalene & $0(12)$ & 12 & $51(97)$ \\
9 & 1-octene & 33 & 33 & $92(176)$ \\
10 & 2-octene & 41 & 41 & $112(214)$ \\
11 & 1-octyne & $21(50)$ & 72 & $253(482)$ \\
12 & 4-octyne & $31(14)$ & 45 & $129(245)$ \\
13 & 1,3-cyclohexadiene & $20(6)$ & 26 & $69(131)$ \\
14 & 1,4 -cyclohexadiene & $22(3)$ & 26 & $59(112)$ \\
\hline
\end{tabular}

a In case of triple bonds the product of single hydrogenation is the $Z$-isomer, exclusively; ${ }^{\mathrm{b}}$ The TON in brackets is considered with the magic number approach, with a particle size of $2.0 \mathrm{~nm}$ [35,37] which corresponds to $52.6 \%$ surface atoms. Each reaction has been performed at least twice.

Among the tested compounds, simple alkenes and alkynes showed the highest conversion upon use of the Ru@[BMMIM][NTf2] system. The hydrogenation of cyclohexene succeeds with mediocre TONs (Table 2; entry 1). 1,5-cyclooctadiene can be hydrogenated with moderate TONs of 253 considering only available surface atoms, where the single hydrogenation is strongly favoured over the full hydrogenation (Table 2; entry 2; 55\%:5\%). The hydrogenation of 1-octyne shows an inverse selectivity (21\%:50\%) and also the highest activity observed overall (Table 2, entry 11; TON 482). In comparison 4-octyne is hydrogenated, but to a smaller extent (TON 245) and less selective (Table 2, entry 12; 31\%:14\%), probably due to the higher steric hindrance. The alkynes phenylacetylene and diphenylacetylene are slowly converted with TONs of 50 and 45, respectively, and selective towards the corresponding alkenes are observed (Table 2, entries 5-6). In case of alkenes, the highest conversion is observed with styrene (Table 2, entry 7; TON 241). Squalene, 1-octene, 2-octene as well as 1, 3- and 1,4-cyclohexadiene are hydrogenated slightly slower (Table 2; entries 8-10 and 13-14). While benzonitrile (Table 2, entry 3 ) remains unchanged, nitrobenzene (Table 2, entry 4 ) can be converted to 
aniline with a TON of 205. No hydrogenation of arenes was observed for these functionalised arenes, which stays in agreement with earlier results that employed elemental hydrogen as the reducing agent for functionalised and unfunctionalized arenes [35-37,41].

Moreover, palladium nanoparticles in the IL $[(\mathrm{BCN}) \mathrm{MIM}]\left[\mathrm{NTf}_{2}\right]$ have been evaluated for transfer hydrogenation from EDAB to organic substrates containing alkynes and alkenes (Table 3).

Table 3. Catalyzed transfer hydrogenations with EDAB and $\operatorname{Pd@}[(\mathrm{BCN}) \mathrm{MIM}]\left[\mathrm{NTf}_{2}\right]$ at $70{ }^{\circ} \mathrm{C}$ after $18-24 \mathrm{~h}$.

\begin{tabular}{ccccc}
\hline$\#$ & Substrate & {$\left[\mathbf{\%}^{\mathbf{a}}\right.$} & {$[\mathbf{\%}]$ Overall } & $\mathbf{T O N}^{\mathbf{b}}$ \\
\hline 1 & phenylacetylene & $9.5(8.1)$ & 18 & $122(665)$ \\
2 & diphenylacetylene & $54-69(1-9)$ & $64-71$ & $186-216(1020-1182)$ \\
3 & 1-octyne & $28(32)$ & 59 & $449(2453)$ \\
4 & 4-octyne & 54 & 11 & $400(2186)$ \\
5 & 1-octene & 45 & 45 & $255(1395)$ \\
6 & 2-octene & 37 & 37 & $245(1337)$ \\
7 & styrene & 47 & 47 & $316(1727)$ \\
8 & cyclohexene & 34 & 34 & $199(1090)$ \\
9 & benzonitrile & 0 & 0 & 0.0 \\
10 & squalene & traces & n.d. & .d. \\
11 & 1,3 -cyclohexadiene & $42(0.2)$ & 42 & $197(1075)$ \\
12 & $1,4-$ cyclohexadiene & $43(3)$ & 46 & $229(1253)$ \\
13 & 1,5 -cyclooctadiene & 4 & 0.2 & $19(104)$ \\
\hline
\end{tabular}

a In case of triple bonds the product of single hydrogenation is the $Z$-isomer, exclusively; ${ }^{\mathrm{b}}$ The TON (turnover number) in brackets is considered with the magic number approach, with a particle size of $7.3 \mathrm{~nm}$ [39], which corresponds to $18.3 \%$ surface atoms. Each reaction has been performed at least twice. n.d.: not determined.

The hydrogenation of phenylacetylene (Table 3, entry 1) proceeds with overall small yields and low selectivity. Interestingly, diphenylacetylene is hydrogenated somewhat faster, despite the higher steric demand (Table 3, entry 2). A strong selectivity towards the single hydrogenated $Z$-alkene is observed (69\%:1\%). The hydrogenation of 1- and 4-octyne (Table 3, entries 3-4) succeeds with TONs of 2453 and 2186, respectively. Selectivity differs strongly among these substrates: the fully hydrogenated product of 1-octyne is found in higher abundance (32\% of $59 \%$ overall yield) than that of 4-octyne (entry 4 , $11 \%$ of $65 \%$ overall yield). Simple alkenes, i.e., 1-octene, 2 -octene, styrene, and cyclohexene (Table 3, entries 5-8), are hydrogenated with moderate TONs of 1090-1727. No hydrogenation of arenes is observed. Similarly to the reaction employing the previously described Ru-system, nitriles are not hydrogenated (Table 3, entry 9). Squalene (Table 3, entry 10) yields only product traces and the reduction of nitrobenzene proceeds uncontrolled and was not further examined. The dienes 1,3- and 1,4cyclohexadiene (CHD) (Table 3, entries 11-12; CHD) can be hydrogenated with similar TONs (1075-1253) and selectivities (1,3-CHD: $0.2 \%$ cyclohexane of $42 \%$ overall yield and 1,4-CHD: $3 \%$ cyclohexane of $46 \%$ overall yield). Hydrogenation of 1,5-cyclooctadiene (Table 3, entry 13) yields only minor amounts of product (entry 13,4\% cyclooctene of $4.2 \%$ overall yield). Conclusively, the substrates accessible to transfer-hydrogenation with $\mathrm{Pd} @[(\mathrm{CN}) \mathrm{BMIM}]\left[\mathrm{NTf}_{2}\right]$ using EDAB are very similar to those of the Ru-system previously described. In most cases, the Pd-system shows a higher activity and a better selectivity towards the partial hydrogenated product (Table 4). In the reduction of 1,5-cyclooctadiene 
and squalene, the Ru-system showed a considerably higher activity. The reason for these exemptions is yet to be found.

Table 4. Comparison between Ru-NPs and Pd-NPs.

\begin{tabular}{|c|c|c|c|c|c|c|c|c|c|}
\hline & \multirow{3}{*}{ Substrate } & \multirow{3}{*}{ Sing. Transf. } & \multirow{3}{*}{ Mult. Transf. } & \multicolumn{3}{|c|}{ Ru-NP@(IL-1) } & \multicolumn{3}{|c|}{ Pd-NP@(24) or @(IL-2) } \\
\hline & & & & \multirow{2}{*}{ TON } & \multicolumn{2}{|c|}{ Conv. [\%] } & \multirow{2}{*}{ TON } & \multicolumn{2}{|c|}{ Conv. [\%] } \\
\hline & & & & & Sing. & Mult. & & Sing. & Mult. \\
\hline 1 & & & 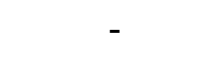 & 211 & 25 & - & 1108 & 2.70 & 34 \\
\hline 2 & & & & 392 & 55 & 5 & 106 & $4^{\mathrm{a}}$ & 0 \\
\hline 3 & & & & 0 & - & 0 & 0 & - & 0 \\
\hline 4 & & & & 318 & - & 17 & & gration & \\
\hline 5 & & & & 147 & 8 & 8 & 676 & 10 & 8 \\
\hline 6 & & & & 133 & 22 & 5 & 1202 & 69 & 2 \\
\hline 7 & & & - & 372 & 45 & - & 1756 & 47 & - \\
\hline 8 & Squalene & & Squalane & 151 & - & 12 & n.d. & - & traces \\
\hline 9 & & & - & 272 & 33 & - & 1418 & 45 & - \\
\hline 10 & & & - & 331 & 41 & - & 1359 & 37 & - \\
\hline 11 & & & & 745 & 21 & 50 & 2494 & 28 & 32 \\
\hline 12 & & & & 0 & 31 & 14 & 2223 & 54 & 10 \\
\hline 13 & & & & 380 & 20 & 6 & 1092 & 42 & 0 \\
\hline 14 & & & & 202 & 22 & 3 & 1274 & 43 & 0 \\
\hline
\end{tabular}

${ }^{\mathrm{a}}[(\mathrm{BCN}) \mathrm{M} 2 \mathrm{Im}][\mathrm{NTf2}]$ was used.

\section{Experimental Section}

\subsection{General}

All commercial chemicals and solvents were purchased from Sigma-Aldrich (St. Louis, MO, USA), Merck (Kenilworth, NJ, USA) and Acros (Waltham, MA, USA) and used as received. Ethylene diamine bisborane (EDAB) [26,27,31], all used ionic liquids [39,52], ruthenium nanoparticles [37], and palladium nanoparticles [39] were prepared using previously published procedures. Nuclear magnetic resonance spectroscopy was performed at $300 \mathrm{~K}$ using a Bruker Avance II 300 or a Bruker Avance II 200 by Bruker (Billerica, MA, USA) at given frequency: AvanceII $300\left({ }^{1} \mathrm{H}(300.1 \mathrm{MHz}),{ }^{13} \mathrm{C}(75.5 \mathrm{MHz})\right.$, $\left.{ }^{11} \mathrm{~B}(96.2 \mathrm{MHz}),{ }^{19} \mathrm{~F}(282.2 \mathrm{MHZ})\right)$, and AvanceII $200\left({ }^{1} \mathrm{H}(200.1 \mathrm{MHz}){ }^{13} \mathrm{C}(50.0 \mathrm{MHz})\right)$. If not otherwise indicated, all measurements were proton broad band-decoupled. The relative chemical shift $\delta$ is 
referenced in the remaining signal of the given deuterated solvent for ${ }^{1} \mathrm{H}$ and ${ }^{13} \mathrm{C}$ spectra. For ${ }^{11} \mathrm{~B}$ spectra, $\mathrm{BF}_{3}$-diethylether complex was added as standard in sealed glass ampules. For ${ }^{19} \mathrm{~F}$ spectra, trifluoromethane $(\delta=0 \mathrm{ppm})$ was used as internal standard, respectively. All chemical shifts are given in parts per million ( $\mathrm{ppm}$ ). When possible, the multiplicity of each spectrum is assigned using the following abbreviations: s: singlet, d: doublet, t: triplet, q: quartet quint: quintet, sext: sextet, sept: septet, m: multiplet, br: broad signal, and mc: multiplet, centred. GC-MS was performed on an Agilent GC-system series 6890 by Agilent using a capillary column HP5MS- $-0.25 \mu \mathrm{m}$ of $30 \mathrm{~m}$ length and $0.32 \mathrm{~mm}$ in diameter by J \& W (J \& W Scientific, branch of Agilent, Santa Clara, CA, USA). The used temperature program $50-300 \mathrm{M}$ had a temperature increase of $10{ }^{\circ} \mathrm{C} / \mathrm{min}$ with a starting temperature of $50{ }^{\circ} \mathrm{C}$, an ending temperature of $300^{\circ} \mathrm{C}$, and a run time of $5 \mathrm{~min}$. Mass detection was carried out on an Agilent 5973 Network Mass Selective Detector by Agilent. Ionization was achieved by electron impact ionization (EI) with an ionization potential of $70 \mathrm{eV}$.

\subsection{Experimental Protocols}

\subsubsection{Nanoparticle Synthesis}

Ruthenium Nanoparticle Synthesis [35,37]

The synthesis of Ru-NPs was carried out according to literature-reported procedures [35,37]. Under glove box conditions, a screw neck vial was loaded with approximately 5-15 $\mathrm{mg}$ of bis(2-methylallyl)(1,5-cyclooctadiene)ruthenium(II) and 0.65-1.45 $\mathrm{mg}$ of ionic liquid [BMMIM] $\left.\mathrm{NTf}_{2}\right]$ and sealed. The reaction mixture was stirred $18 \mathrm{~h}$ at $75^{\circ} \mathrm{C}$ to yield a brown to dark brown but clear suspension. The obtained particle size averaged $2.0 \mathrm{~nm}$ with a size distribution of $\pm 0.5 \mathrm{~nm}[35,37]$.

Palladium Nanoparticle Synthesis [39]

The synthesis of Pd-NPs was carried out according to a literature-reported procedure [39]. A screw neck vial was loaded with approximately $3 \mathrm{mg}$ of palladium(II)acetate and $600 \mathrm{mg}$ of ionic liquid [(BCN)MIM] $\left[\mathrm{NTf}_{2}\right]$ and sealed. The reaction mixture was stirred $3 \mathrm{~h}$ at $130{ }^{\circ} \mathrm{C}$ to yield an orange but clear suspension. The obtained particle size averaged $7.1 \mathrm{~nm}$ with a size distribution of $\pm 2.2 \mathrm{~nm}$ [39].

\subsubsection{Metal-Free Transfer Hydrogenation in Water}

Exemplary procedure (Table 1; entry 2): A vial was charged with $2.3 \mathrm{mmol}$ acetophenone (Table 1) and $5 \mathrm{~mL}$ demineralized water. After adding $0.5 \mathrm{mmol}$ of EDAB (four $\mathrm{H}_{2}$ equivalents), the vial was sealed, placed in an aluminum heating block at $80{ }^{\circ} \mathrm{C}$, and the mixture was stirred for the given time (Table 1). Subsequently, the aqueous suspension was cooled down and extracted three times with $5 \mathrm{~mL}$ $\mathrm{Et}_{2} \mathrm{O}$. The organic layers were combined and the solvent was removed under reduced pressure. The product yield was determined by NMR spectroscopy using a defined amount of $\mathrm{Si}_{2} \mathrm{Me}_{6}$ as internal standard.

\subsubsection{Transfer Hydrogenations in Ionic Liquids with Metal Nanoparticles}

Exemplary procedure (Table 2; entry 1): In a typical transfer hydrogenation experiment, a vial was charged with the ruthenium nanocatalyst mixture of previously synthesized ruthenium nanoparticles 
(3 mg Ru) in ionic liquid (1.28 g; Ru@BMMIM][NTf2]) [37] and 9 mmol cyclohexene. Subsequently, $1.7 \mathrm{mmol}$ EDAB was added and the vial was sealed. The reaction mixture was stirred overnight at $70{ }^{\circ} \mathrm{C}$ and then cooled to room temperature. A defined amount of $\mathrm{CDCl}_{3}$ as solvent and hexamethyl disilane (HMDS; $\mathrm{Si}_{2} \mathrm{Me}_{6}$ ) as internal standard was added to the mixture and homogenized. A sample was taken and the product yield was determined by NMR spectroscopy (Tables 2 and 3). The $\mathrm{Pd}$-catalyzed reactions used $600 \mathrm{mg}[(\mathrm{CN}) \mathrm{BMIM}]\left[\mathrm{NTf}_{2}\right]$ and $0.5 \mathrm{mg} \mathrm{Pd}$ nanoparticles prepared as previously published [39].

\section{Conclusions}

In summary, ethylenediamine bisborane (EDAB) is a suitable hydrogen source for transfer hydrogenation reactions on $\mathrm{C}-\mathrm{C}$ double bonds mediated by metal nanoparticles. Several organic model compounds could be shown to undergo reduction in the presence of the Ru-system as well as the Pd-system. In most cases, the latter showed considerably higher activity. The single hydrogenation of triple bonds yields only $Z$-isomeric compounds, as expected in a heterogeneous catalytic reaction. C-N triple bonds (nitriles) are not susceptible to the reduction. $\mathrm{C}-\mathrm{O}$ double bonds can be reduced without the presence of metal particles in water. $\mathrm{C}$-C double bonds can be readily reduced, while aromatic double bonds remain inert. In future investigations, the selectivity, time-resolved studies, and solvent effects will be addressed. The reduction with this air- and moisture-stable amine borane adduct could complement the synthetically interested chemist's toolkit.

\section{Acknowledgments}

We acknowledge the Ministerium für Innovation, Wissenschaft und Forschung NRW (MIWF-NRW) for financial support within the Energy Research Program for the Scientist Returnee Award for M.H.G. Prechtl (NRW-Rückkehrerprogramm). M.H.G.P. acknowledges the Ernst-Haage Foundation at the Max-Planck-Institute for Chemical Energy Conversion for the Ernst-Haage Prize 2014 and the Deutsche Forschungsgemeischaft DFG (Heisenberg-program). S.S., M.S., and C.G. want to thank M. Kessler, L. Heim, and H. Konnerth for helpful discussion.

\section{Author Contributions}

S.S., M.S. and C.G. performed experiments and analyses. S.S., M.S., and M.H.G.P. wrote the manuscript.

\section{Conflicts of Interest}

The authors declare no conflict of interest.

\section{References}

1. Stanislaus, A.; Cooper, B.H. Aromatic hydrogenation catalysis: A review. Catal. Rev. 1994, 36, 75-123.

2. Jessop, P.G.; Ikariya, T.; Noyori, R. Homogeneous hydrogenation of carbon dioxide. Chem. Rev. 1995, 95, 259-272. 
3. Blaser, H.-U.; Malan, C.; Pugin, B.; Spindler, F.; Steiner, H.; Studer, M. Selective hydrogenation for fine chemicals: Recent trends and new developments. Adv. Synth. Catal. 2003, 345, 103-151.

4. Palmer, M.J.; Wills, M. Asymmetric transfer hydrogenation of $\mathrm{C}=\mathrm{O}$ and $\mathrm{C}=\mathrm{N}$ bonds. Tetrahedron Asymmetry 1999, 10, 2045-2061.

5. Johnstone, R.A.W.; Wilby, A.H.; Entwistle, I.D. Heterogeneous catalytic transfer hydrogenation and its relation to other methods for reduction of organic compounds. Chem. Rev. 1985, 85, 129-170.

6. Gallezot, P.; Richard, D. Selective hydrogenation of $\alpha, \beta$-unsaturated aldehydes. Catal. Rev. 1998, 40, 81-126.

7. Noyori, R.; Hashiguchi, S. Asymmetric transfer hydrogenation catalyzed by chiral ruthenium complexes. Acc. Chem. Res. 1997, 30, 97-102.

8. Rana, M.S.; Sámano, V.; Ancheyta, J.; Diaz, J.A.I. A review of recent advances on process technologies for upgrading of heavy oils and residua. Fuel 2007, 86, 1216-1231.

9. Ward, J.W. Hydrocracking processes and catalysts. Fuel Process. Technol. 1993, 35, 55-85.

10. Erisman, J.W.; Sutton, M.A.; Galloway, J.; Klimont, Z.; Winiwarter, W. How a century of ammonia synthesis changed the world. Nat. Geosci. 2008, 1, 636-639.

11. Fouilloux, P. The nature of raney nickel, its adsorbed hydrogen and its catalytic activity for hydrogenation reactions (review). Appl. Catal. 1983, 8, 1-42.

12. Samec, J.S.; Backvall, J.E.; Andersson, P.G.; Brandt, P. Mechanistic aspects of transition metal-catalyzed hydrogen transfer reactions. Chem. Soc. Rev. 2006, 35, 237-248.

13. Göksu, H.; Ho, S.F.; Metin, Ö.; Korkmaz, K.; Mendoza Garcia, A.; Gültekin, M.S.; Sun, S. Tandem dehydrogenation of ammonia borane and hydrogenation of nitro/nitrile compounds catalyzed by graphene-supported nipd alloy nanoparticles. ACS Catal. 2014, 4, 1777-1782.

14. Clapham, S.E.; Hadzovic, A.; Morris, R.H. Mechanisms of the h2-hydrogenation and transfer hydrogenation of polar bonds catalyzed by ruthenium hydride complexes. Coord. Chem. Rev. 2004, 248, 2201-2237.

15. Gladiali, S.; Alberico, E. Asymmetric transfer hydrogenation: Chiral ligands and applications. Chem. Soc. Rev. 2006, 35, 226-236.

16. Morris, R.H. Asymmetric hydrogenation, transfer hydrogenation and hydrosilylation of ketones catalyzed by iron complexes. Chem. Soc. Rev. 2009, 38, 2282-2291.

17. Yamakawa, M.; Ito, H.; Noyori, R. The metal—Ligand bifunctional catalysis: A theoretical study on the ruthenium(ii)-catalyzed hydrogen transfer between alcohols and carbonyl compounds. J. Am. Chem. Soc. 2000, 122, 1466-1478.

18. Fujii, A.; Hashiguchi, S.; Uematsu, N.; Ikariya, T.; Noyori, R. Ruthenium(ii)-catalyzed asymmetric transfer hydrogenation of ketones using a formic acid-Triethylamine mixture. J. Am. Chem. Soc. 1996, 118, 2521-2522.

19. Zheng, C.; You, S.L. Transfer hydrogenation with hantzsch esters and related organic hydride donors. Chem. Soc. Rev. 2012, 41, 2498-2518.

20. Knowles, W.S. Asymmetric hydrogenation. Acc. Chem. Res. 1983, 16, 106-112.

21. Alonso, D.A.; Brandt, P.; Nordin, S.J.M.; Andersson, P.G. Ru(arene)(amino alcohol)-catalyzed transfer hydrogenation of ketones: Mechanism and origin of enantioselectivity. J. Am. Chem. Soc. 1999, 121, 9580-9588.

22. List, B. Asymmetric aminocatalysis. Synlett 2001, 2001, 1675-1686. 
23. Paras, N.A.; MacMillan, D.W.C. New strategies in organic catalysis: The first enantioselective organocatalytic friedel-Crafts alkylation. J. Am. Chem. Soc. 2001, 123, 4370-4371.

24. Staubitz, A.; Robertson, A.P.; Manners, I. Ammonia-borane and related compounds as dihydrogen sources. Chem. Rev. 2010, 110, 4079-4124.

25. Bowden, M.E.; Brown, I.W.M.; Gainsford, G.J.; Wong, H. Structure and thermal decomposition of methylamine borane. Inorg. Chim. Acta 2008, 361, 2147-2153.

26. Sahler, S.; Konnerth, H.; Knoblauch, N.; Prechtl, M.H.G. Hydrogen storage in amine boranes: Ionic liquid supported thermal dehydrogenation of ethylene diamine bisborane. Int. J. Hydrog. Energy 2013, 38, 3283-3290.

27. Neiner, D.; Karkamkar, A.; Bowden, M.; Joon Choi, Y.; Luedtke, A.; Holladay, J.; Fisher, A.; Szymczak, N.; Autrey, T. Kinetic and thermodynamic investigation of hydrogen release from ethane 1,2-di-amineborane. Energy Environ. Sci. 2011, 4, 4187-4193.

28. Yang, X.; Fox, T.; Berke, H. Facile metal free regioselective transfer hydrogenation of polarized olefins with ammonia borane. Chem. Commun. 2011, 47, 2053-2055.

29. Yang, X.; Fox, T.; Berke, H. Ammonia borane as a metal free reductant for ketones and aldehydes: A mechanistic study. Tetrahedron 2011, 67, 7121-7127.

30. Yang, X.; Zhao, L.; Fox, T.; Wang, Z.X.; Berke, H. Transfer hydrogenation of imines with ammonia-borane: A concerted double-hydrogen-transfer reaction. Angew. Chem. 2010, 49, 2058-2062.

31. Sahler, S.; Sturm, S.; Kessler, M.T.; Prechtl, M.H.G. The role of ionic liquids in hydrogen storage. Chem. Eur. J. 2014, 20, 8934-8941.

32. Anastas, P.T.; Zimmerman, J.B. Peer reviewed: Design through the 12 principles of green engineering. Environ. Sci. Technol. 2003, 37, 94A-101A.

33. Curzons, A.D.; Mortimer, D.N.; Constable, D.J.C.; Cunningham, V.L. So you think your process is green, how do you know?-Using principles of sustainability to determine what is green-A corporate perspective. Green Chem. 2001, 3, 1-6.

34. Dupont, J.; de Souza, R.F.; Suarez, P.A. Ionic liquid (molten salt) phase organometallic catalysis. Chem. Rev. 2002, 102, 3667-3692.

35. Prechtl, M.H.G.; Scariot, M.; Scholten, J.D.; Machado, G.; Teixeira, S.R.; Dupont, J. Nanoscale $\mathrm{Ru}(0)$ particles: Arene hydrogenation catalysts in imidazolium ionic liquids. Inorg. Chem. 2008, 47, 8995-9001.

36. Prechtl, M.H.G.; Scholten, J.D.; Dupont, J. Tuning the selectivity of ruthenium nanoscale catalysts with functionalised ionic liquids: Hydrogenation of nitriles. J. Mol. Catal. A-Chem. 2009, 313, 74-78.

37. Prechtl, M.H.G.; Campbell, P.S.; Scholten, J.D.; Fraser, G.B.; Machado, G.; Santini, C.C.; Dupont, J.; Chauvin, Y. Imidazolium ionic liquids as promoters and stabilising agents for the preparation of metal( 0$)$ nanoparticles by reduction and decomposition of organometallic complexes. Nanoscale 2010, 2, 2601-2606.

38. Prechtl, M.H.G.; Scholten, J.D.; Dupont, J. Carbon-carbon cross coupling reactions in ionic liquids catalysed by palladium metal nanoparticles. Molecules 2010, 15, 3441-3461. 
39. Venkatesan, R.; Prechtl, M.H.G.; Scholten, J.D.; Pezzi, R.P.; Machado, G.; Dupont, J. Palladium nanoparticle catalysts in ionic liquids: Synthesis, characterisation and selective partial hydrogenation of alkynes to z-alkenes. J. Mater. Chem. 2011, 21, 3030-3036.

40. Keßler, M.T.; Gedig, C.; Sahler, S.; Wand, P.; Robke, S.; Prechtl, M.H.G. Recyclable nanoscale copper(i) catalysts in ionic liquid media for selective decarboxylative c-c bond cleavage. Catal. Sci. Technol. 2013, 3, 992-1001.

41. Darwich, W.; Gedig, C.; Srour, H.; Santini, C.C.; Prechtl, M.H.G. Single step synthesis of metallic nanoparticles using dihydroxyl functionalized ionic liquids as reductive agent. $R S C A d v . \mathbf{2 0 1 3}, 3$, 20324.

42. Yan, J.M.; Zhang, X.B.; Akita, T.; Haruta, M.; Xu, Q. One-step seeding growth of magnetically recyclableau@co core-shell nanoparticles: Highly efficient catalyst for hydrolytic dehydrogenation of ammonia borane. J. Am. Chem. Soc. 2010, 132, 5326-5327.

43. Metin, O.; Mazumder, V.; Ozkar, S.; Sun, S. Monodisperse nickel nanoparticles and their catalysis in hydrolytic dehydrogenation of ammonia borane. J. Am. Chem. Soc. 2010, 132, 1468-1469.

44. Chandra, M.; Xu, Q. Room temperature hydrogen generation from aqueous ammonia-borane using noble metal nano-clusters as highly active catalysts. J. Power Source 2007, 168, 135-142.

45. Yan, J.M.; Zhang, X.B.; Han, S.; Shioyama, H.; Xu, Q. Iron-nanoparticle-catalyzed hydrolytic dehydrogenation of ammonia borane for chemical hydrogen storage. Angew. Chem. 2008, 47, 2287-2289.

46. Alonso, F.; Riente, P.; Yus, M. Nickel nanoparticles in hydrogen transfer reactions. Acc. Chem. Res. 2011, 44, 379-391.

47. Sonnenberg, J.F.; Coombs, N.; Dube, P.A.; Morris, R.H. Iron nanoparticles catalyzing the asymmetric transfer hydrogenation of ketones. J. Am. Chem. Soc. 2012, 134, 5893-5899.

48. Andrews, G.C. Chemoselectivity in the reduction of aldehydes and ketones with amine boranes. Tetrahedron Lett. 1980, 21, 697-700.

49. Andrews, G.C.; Crawford, T.C. Synthetic utility of amine borane reagents in the reduction of aldehydes and ketones. Tetrahedron Lett. 1980, 21, 693-696.

50. Shi, L.; Liu, Y.Y.; Liu, Q.F.; Wei, B.; Zhang, G.S. Selective reduction of aldehydes and ketones to alcohols with ammonia borane in neat water. Green Chem. 2012, 14, 1372-1375.

51. Duan, Y.F.; Bai, R.J.; Tian, J.; Chen, L.G.; Yan, X.L. Hydrogenation of aldehydes and ketones to corresponding alcohols with methylamine borane in neat water. Synth. Commun. 2014, 44, 2555-2564.

52. Cassol, C.C.; Ebeling, G.; Ferrera, B.; Dupont, J. A simple and practical method for the preparation and purity determination of halide-free imidazolium ionic liquids. Adv. Synth. Catal. 2006, 348, 243-248.

Sample Availability: Not applicable; no new compounds have been synthesised.

(C) 2015 by the authors; licensee MDPI, Basel, Switzerland. This article is an open access article distributed under the terms and conditions of the Creative Commons Attribution license (http://creativecommons.org/licenses/by/4.0/). 